\title{
Plasmon-induced nonlinearity enhancement and homogenization of graphene metasurfaces
}

\author{
Jian Wei You ${ }^{1}$ and Nicolae C. Panoiu ${ }^{1, *}$ \\ ${ }^{1}$ Department of Electronic and Electrical Engineering, University College London, London, WC1E 7JE, UK \\ *Corresponding author: n.panoiu@ucl.ac.uk

We demonstrate that the effective third-order nonlinear susceptibility of a graphene sheet can be enhanced by more than two orders of magnitude by patterning it into a graphene metasurface. In addition, in order to gain deeper physical insights into this phenomenon, we introduce a versatile homogenization method, which is subsequently used to characterize quantitatively this nonlinearity enhancement effect by calculating the effective linear and nonlinear susceptibility of graphene metasurfaces. The accuracy of the proposed homogenization method is demonstrated by comparing its predictions with those obtained from the Kramers-Kronig relations. This work may open up new opportunities to explore novel physics pertaining to nonlinear optical interactions in graphene metasurfaces. () 2019 Optical Society of America

OCIS codes: (160.3918) Metamaterials; (160.4330) Nonlinear Optical Materials; (190.4360) Nonlinear Optics, Devices.

http://dx.doi.org/10.1364/ao.XX.XXXXXX

Since it has been first isolated from graphite [1], the unique and striking properties of graphene have spurred intense research efforts to develop and synthesize new two-dimensional (2D) materials. So far, graphene and other 2D materials have already had a great impact both as facilitators of key advancements in fundamental research, as well as enablers of new devices operating in a broad spectrum, ranging from ultraviolet, visible and down to microwave frequencies [2-8]. For instance, due to their unique linear physical properties, 2D materials have found important applications to electronics [5, 6], sensors [7], and solar cells [8]. Equally important, the nonlinear optical properties of graphene have facilitated the development of new active photonic devices with improved functionality [9-12] and the exploration in new physical conditions of fundamental phenomena, including spatial solitons [11] and tunable Dirac points [12].

Inspired by the concept of metasurfaces, the research in graphene has expanded from the study of uniform monolayer configurations to nanopatterned surface structures. These graphene metasurfaces have open up promising new routes towards photonic devices with specially engineered linear and nonlinear optical responses [13-20]. A key role in these developments has been played by versatile and powerful numerical and analytical methods. In particular, the use of homogenization methods that reduce the optical response of a metasurface to that of a homogeneous layer of material characterized by specific optical constants is ubiquitous in the design process and analysis of metasurfaces. Two of the most common homogenization methods are the scattering-parameter approach [21] and the field-averaging procedure [22]. To date, they have been applied mostly to metasurfaces containing linear, dispersive, and isotropic materials, with scarce efforts being devoted to the nonlinear case $[23,24]$. One of the main reasons for this is that the high-order nonlinear susceptibilities of graphene are anisotropic, which significantly hinders the extension of the existing linear homogenization methods to the nonlinear case.

In this Letter, we propose a versatile linear and nonlinear homogenization method for metasurfaces containing graphene. The challenges arisen from the nonlinear and anisotropic characteristics of such metasurfaces are overcome by introducing a set of auxiliary physical quantities, which allows one to unambiguously match the far-field optical response of the metasurface with that of a homogeneous layer of material with certain constitutive parameters. This versatile homogenization method is used to study the linear and nonlinear optical properties of a generic graphene metasurface. Our study reveals that, at the resonance frequencies of surface plasmons of nano-sized graphene constituents of the metasurface the effective third-order susceptibility of the metasurface is enhanced by more than two orders of magnitude as compared to that of a graphene sheet.

The schematics of the graphene metasurface investigated in this work is presented in Fig. 1(a), with the unit cell depicted in Fig. 1(b). The metasurface lies in the $x-y$ plane, and consists of a rectangular array of cruciform graphene patches. The symmetry axes of the array and cruciform patches are along the $x$ - and $y$-axes, the corresponding periods being $P_{x}$ and $P_{y}$. The length and width of the arms of the crosses are $\left(L_{x}, L_{y}\right)$ and $\left(W_{x}, W_{y}\right)$, respectively. Unless otherwise specified, the values of these parameters are $P_{x}=P_{y}=200 \mathrm{~nm}, L_{x}=L_{y}=180 \mathrm{~nm}$, and $W_{x}=W_{y}=75 \mathrm{~nm}$. Moreover, the relative electric permittivity of graphene is $\varepsilon_{g}=1+i \sigma_{s} /\left(\varepsilon_{0} \omega h_{g}\right)$, where $h_{g}=0.5 \mathrm{~nm}$ is the thickness of graphene, $\omega$ is the frequency, and the graphene surface conductivity, $\sigma_{S}$, is described by the Kubo's formula [25]:

$$
\sigma_{S}=\frac{e^{2} k_{B} T \tau}{\pi \hbar^{2} \bar{\omega}}\left[\frac{\mu_{c}}{k_{B} T}+2 \ln \left(e^{-\frac{\mu_{c}}{k_{B} T}}+1\right)\right]+\frac{i e^{2}}{4 \pi \hbar} \ln \frac{\xi-i \bar{\omega}}{\xi+i \bar{\omega}} .
$$

Here, $\mu_{c}, T$, and $\tau$ are the chemical potential, temperature, and 


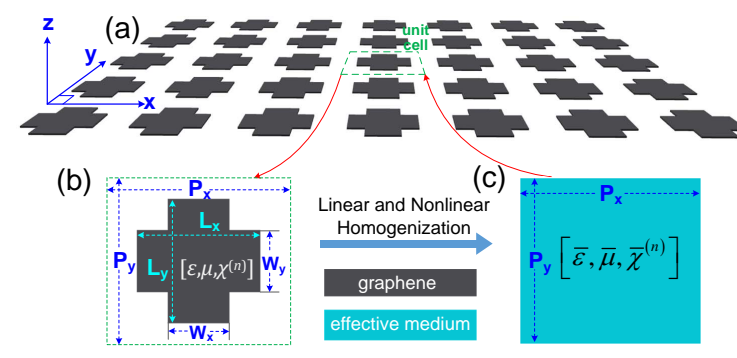

Fig. 1. (a) Schematics of a graphene-based metasurfaces consisting of a rectangular array of graphene cruciform patches. (b) Geometry and material parameters size of a unit cell of the metasurface. (c) The homogenized metasurface, characterized by effective linear and nonlinear optical constants.

relaxation time, respectively, $\bar{\omega}=1-i \omega \tau$, and $\xi=2\left|\mu_{c}\right| \tau / \hbar$. In this study, we use $\mu_{c}=0.2 \mathrm{eV}, \tau=0.1 \mathrm{ps}$, and $T=300 \mathrm{~K}$.

Generally, the linear constitutive parameters of a homogenized metasurface are uniquely determined. In the nonlinear case, however, the effective nonlinear susceptibilities of a homogenized metasurface are not uniquely determined as in general there are more independent components of these tensor quantities than available constitutive relations. Our homogenization method circumvents this problem by introducing a set of auxiliary variables that define the linear and nonlinear polarizations. These auxiliary variables, and subsequently the linear and nonlinear effective optical constants of the homogenized metasurface, are determined by requiring that the averaged linear and nonlinear polarizations in the original and homogenized metasurfaces are termwise identical.

The homogenization method presented here consists of two steps: First, a standard field averaging method [22] is used to determine the averaged fields at the fundamental frequency (FF) and the effective electric permittivity. In the second step, the fields at the FF are used to determine the nonlinear polarization and, through a set of auxiliary variables, the effective nonlinear susceptibility of the homogenized metasurface. As nonlinear interaction we choose the third-harmonic generation (THG), but our method can be readily used to study other nonlinear interactions characterized by nonlinear polarizations than can be expressed in terms of the field at the FF.

To begin with, let us consider the constitutive relation in a linear and anisotropic material that relates the electric displacement and the electric field, $D_{i}=\sum_{j} \varepsilon_{i j} E_{j}$, where $\varepsilon_{i j}$ is the permittivity, and $i, j=x, y, z$. The spatial average of the fields $\mathbf{E}$ and $\mathbf{D}$ is defined as:

$$
\begin{aligned}
\overline{\mathbf{E}}(\omega) & =\frac{1}{V} \int_{V} \mathbf{E}(\mathbf{r}, \omega) d \mathbf{r}, \\
\overline{\mathbf{D}}(\omega) & =\frac{1}{V} \int_{V} \mathbf{D}(\mathbf{r}, \omega) d \mathbf{r},
\end{aligned}
$$

where $V$ is the volume of the unit cell. These averaged fields can be used to define the effective permittivity of the homogenized metasurface, $\bar{\varepsilon}_{i}=\bar{D}_{i} / \bar{E}_{i}$.

This approach is only applicable to metasurfaces containing isotropic materials, whose permittivity tensor is diagonal, but it can be readily extended to the more general case of anisotropic structures by introducing a new auxiliary quantity, $d_{i j}=\varepsilon_{i j} E_{j}$. Then, the constitutive relation for anisotropic materials is expressed as $D_{i}=\sum_{j} d_{i j}$. Imposing now the condition that the

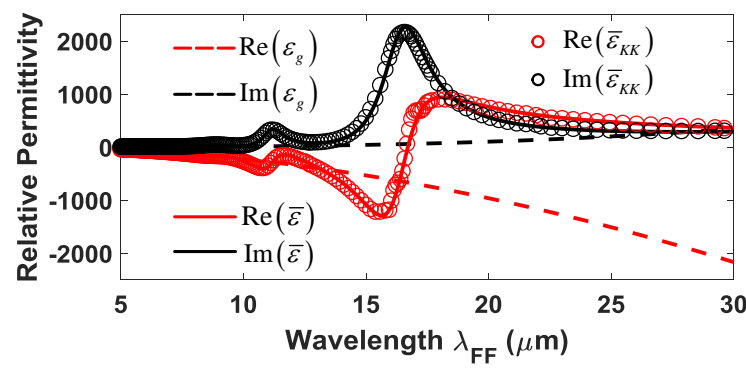

Fig. 2. Wavelength dependence of the real and imaginary parts of graphene permittivity, $\varepsilon_{g}$, the effective permittivity of the homogenized cruciform metasurface, $\bar{\varepsilon}$, and the corresponding permittivity, $\bar{\varepsilon}_{K K}$, calculated from $\bar{\varepsilon}$ using the Kramers-Kronig relations.

averaged electric displacement in the metasurface and the electric displacement in the homogenized metasurface are termwise identical, the effective permittivity tensor can be calculated as:

$$
\bar{\varepsilon}_{i j}(\omega)=\bar{d}_{i j}(\omega) / \bar{E}_{j}(\omega),
$$

where the averaged auxiliary quantity is given by:

$$
\bar{d}_{i j}(\omega)=\frac{1}{V} \int_{V} \varepsilon_{i j}(\mathbf{r}, \omega) E_{j}(\mathbf{r}, \omega) d \mathbf{r} .
$$

The thickness of graphene, and more generally of 2D materials, is much smaller than the optical wavelength at infrared and $\mathrm{THz}$ frequencies, and thus one can assume that the optical field is uniform across graphene. Therefore, the volume integrals in Eq. (2) and Eq. (4) become surface integrals over the mid-section plane of graphene patches.

In order to validate our proposed linear homogenization approach, a graphene cruciform metasurface (see Fig. 1) has been studied using the FDTD method. In the numerical simulations, the graphene cruciform metasurface is illuminated by a normally incident plane wave linearly polarized along the $x$-axis. The relevant results are presented in Fig. 2, where we plot the wavelength dependence of the intrinsic relative permittivity of graphene, the effective permittivity of the homogenized graphene metasurface retrieved from Eq. (3), and the corresponding permittivity determined from the Kramers-Kronig $(\mathrm{KK})$ relations [26], $\bar{\varepsilon}_{K K}$ :

$$
\begin{aligned}
& \mathfrak{R e}\left\{\bar{\varepsilon}_{K K}(\omega)\right\}=1+\frac{2}{\pi} f_{0}^{\infty} \frac{\omega^{\prime} \mathfrak{I m}\left\{\bar{\varepsilon}\left(\omega^{\prime}\right)\right\}}{\omega^{\prime 2}-\omega^{2}} d \omega^{\prime}, \\
& \mathfrak{I m}\left\{\bar{\varepsilon}_{K K}(\omega)\right\}=-\frac{2}{\pi} f_{0}^{\infty} \frac{\omega\left[\mathfrak{R e}\left\{\bar{\varepsilon}\left(\omega^{\prime}\right)\right\}-1\right]}{\omega^{\prime 2}-\omega^{2}} d \omega^{\prime},
\end{aligned}
$$

where the symbol $f$ denotes the Cauchy principal value of the integral. Note that for normal incidence $\bar{\varepsilon}$ does not depend on the polarization angle [27], and $\bar{\varepsilon}_{x x}=\bar{\varepsilon}_{y y}$ and $\bar{\varepsilon}_{x y}=\bar{\varepsilon}_{y x}=0$.

Figure 2 reveals several important properties of the wavelength dispersion of the effective permittivity of the homogenized metasurface. First, it is markedly different from the graphene permittivity and shows a series of Lorentz-type resonances, which correspond to localized surface plasmons of the graphene crosses. Moreover, whereas $\mathfrak{R e}\left(\varepsilon_{g}\right)<0$ in the entire wavelength domain we considered, in some wavelength domains $\mathfrak{R e}(\bar{\varepsilon})>0$. This suggests that at those wavelengths the metasurface responds as a dielectric one. In addition, we note that near the peak $\mathfrak{R e}(\bar{\varepsilon}) \approx 0$ in a narrow frequency range, 


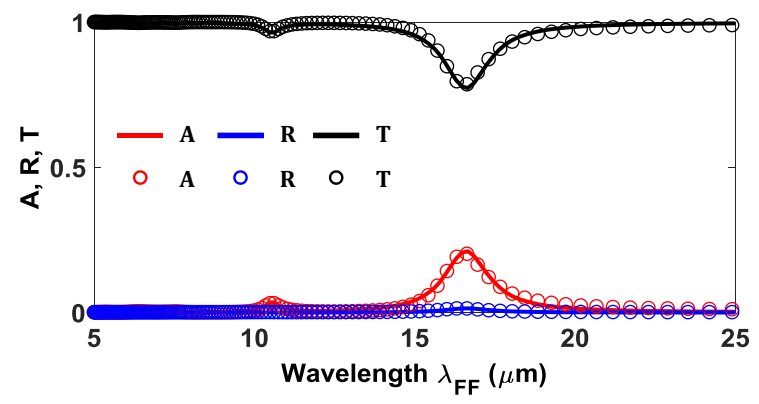

Fig. 3. Comparison between the absorption, $A$, reflectance, $R$, and transmittance, $T$, of the original cruciform graphene metasurface (solid curves) and the homogenized one (circles).

which means that near the resonance the metasurface behaves as an epsilon-near-zero metamaterial. Equally important, it can be seen in Fig. 2 that the permittivity $\bar{\varepsilon}_{K K}$ obtained from the effective permittivity of the metasurface, $\bar{\varepsilon}$, using the KK relations is almost identical with the latter, $\bar{\varepsilon}_{K K} \approx \bar{\varepsilon}$, which validates our method. More specifically, the effective permittivity calculated with our homogenization method obeys the causality principle, proving that it is a physically meaningful quantity.

A key advantage of the homogenization theory is that a metasurface patterned in a convolute manner can be replaced with a simple homogenous layer of a material with specific optical constants in such a way that physical quantities such as absorption, $A$, reflectance, $R$, and transmittance, $T$, of the two optical systems are identical. This provides us an effective tool to validate our homogenization method, namely we quantify the difference between the absorption, reflectance, and transmittance of the original metasurface and the homogenized one. To this end, we have computed these quantities for both optical systems using the FDTD method. The results of these calculations are summarized in Fig. 3. This comparison clearly shows that the linear response of the uniform layer of material with the retrieved effective permittivity is practically the same as that of the graphene cruciform metasurface. This excellent agreement further proves the reliability of our linear homogenization method. Moreover, the data presented in Fig. 3 suggest that the peaks of the absorption spectra shown in this figure coincide with those of the imaginary part of the retrieved permittivity plotted in Fig. 2. This finding is explained by the fact that the peaks in the spectrum of the effective permittivity of the homogenized metasurface correspond to the excitation of localized surface plasmons on the graphene crosses, a phenomenon accompanied by large enhancement of the optical near-field and consequently an increase of the optical absorption.

Encouraged by the accuracy with which our homogenization method describes the linear optical response of graphene metasurfaces, we proceeded to extend it to the much more complex case of nonlinear optical interactions. Due to the centrosymmetric nature of the graphene lattice, the lowest-order non-vanishing nonlinear optical interactions in graphene are of the third-order. In particular, in the case of the third-harmonic generation (THG), the nonlinear polarization is given by:

$$
\mathbf{P}^{n l}(\mathbf{r}, \Omega)=\varepsilon_{0} \chi^{(3)}(\mathbf{r}, \Omega, \omega) \vdots \mathbf{E}(\mathbf{r}, \omega) \mathbf{E}(\mathbf{r}, \omega) \mathbf{E}(\mathbf{r}, \omega),
$$

where $\chi^{(3)}$ is the third-order susceptibility and $\Omega=3 \omega$ is the frequency of the third harmonic (TH). Componentwise, this nonlinear polarization can be written as:

$$
P_{i}^{n l}=\varepsilon_{0} \sum_{j k l} \chi_{i j k l}^{(3)} E_{j} E_{k} E_{l} \equiv \varepsilon_{0} \sum_{j k l} q_{i j k l},
$$

where the nonlinear auxiliary quantities $q_{i j k l}=\chi_{i j k l}^{(3)} E_{j} E_{k} E_{l}$ have been defined. Their averaged values are:

$$
\bar{q}_{i j k l}(\Omega)=\frac{1}{V} \int_{V} \chi_{i j k l}^{(3)}(\mathbf{r}, \Omega, \omega) E_{j}(\mathbf{r}, \omega) E_{k}(\mathbf{r}, \omega) E_{l}(\mathbf{r}, \omega) d \mathbf{r} .
$$

Similarly to Eq. (7), the nonlinear polarization in the homogenized layer can be expressed in terms of the averaged fields at the fundamental frequency (FF) and an effective third-order susceptibility, $\bar{\chi}^{(3)}$, as:

$$
\bar{P}_{i}^{n l}(\Omega)=\varepsilon_{0} \sum_{j k l} \bar{\chi}_{i j k l}^{(3)}(\Omega, \omega) \bar{E}_{j}(\omega) \bar{E}_{k}(\omega) \bar{E}_{l}(\omega) .
$$

Finally, we require that the averaged nonlinear polarizations described by Eq. (7) and Eq. (9) are termwise identical. This ensures that on average the nonlinear polarizations in the graphene metasurface and the homogenized layer of nonlinear material are equal. Under these circumstances, the effective third-order susceptibility, $\bar{\chi}^{(3)}$, is given by the following formula:

$$
\bar{\chi}_{i j k l}^{(3)}(\Omega, \omega)=\frac{\bar{q}_{i j k l}(\Omega)}{\bar{E}_{j}(\omega) \bar{E}_{k}(\omega) \bar{E}_{l}(\omega)} .
$$

We have used this formalism, namely, Eqs. (8) and (10) in conjunction with Eq. (2a), to compute the effective third-order susceptibility $\bar{\chi}^{(3)}$ of the graphene cruciform metasurface, and summarize the relevant results in Fig. 4. The third-order susceptibility of a homogeneous graphene sheet is $\chi_{g}^{(3)}(3 \omega ; \omega)=$ $\left[i /\left(3 \omega \varepsilon_{0} h_{g}\right)\right] \sigma_{s}^{(3)}(3 \omega ; \omega)$, where the third-order conductivity is given by $\sigma_{s, i j k l}^{(3)}(3 \omega ; \omega)=\sigma_{s}^{(3)}\left(\delta_{i j} \delta_{k l}+\delta_{i k} \delta_{j l}+\delta_{i l} \delta_{j k}\right) / 3$ [28], where $\delta_{i j}$ is the Kronecker delta and $\sigma_{s}^{(3)}=\frac{i \sigma_{0}\left(\hbar v_{F} e\right)^{2}}{48 \pi(\hbar \omega)^{4}} T\left(\frac{\hbar \omega}{2\left|\mu_{c}\right|}\right)$. Here, $v_{F} \approx c / 300$ is the Fermi velocity, $\sigma_{0}=e^{2} /(4 \hbar)$ is the universal dynamic conductivity of graphene, and $T(x)=$

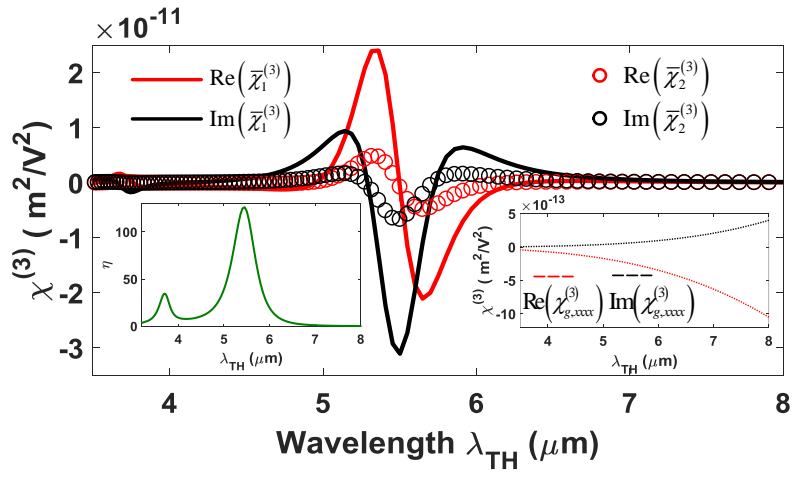

Fig. 4. Wavelength dependence of the two independent components of the effective third-order susceptibility of the graphene metasurface, $\bar{\chi}_{1}^{(3)}$ and $\bar{\chi}_{2}^{(3)}$. Left and right insets, spectra of the enhancement factor $\eta=\left|\bar{\chi}_{1}^{(3)}\right| /\left|\chi_{g}^{(3)}\right|$ and the dominant component of the third-order graphene susceptibility, $\chi_{g}^{(3)} x x x x$, respectively. 


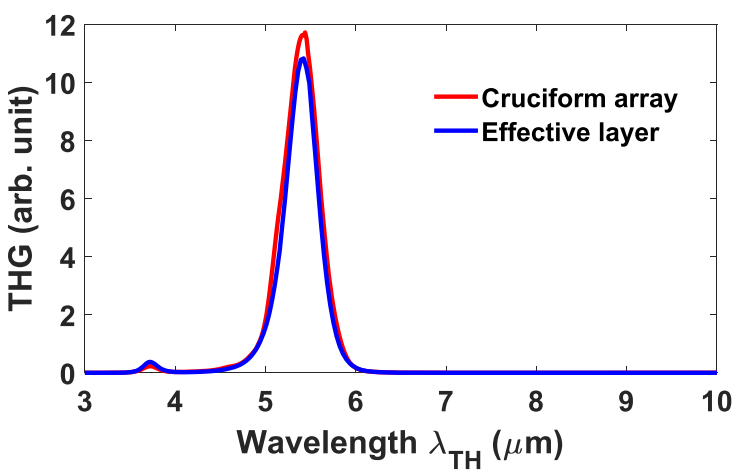

Fig. 5. Third-harmonic generation spectra calculated for a graphene cruciform metasurface and the corresponding homogenized layer of nonlinear optical material.

$17 G(x)-64 G(2 x)+45 G(3 x)$, where $G(x)=\ln \mid(1+x) /(1-$ $x) \mid+i \pi H(|x|-1)$ and $H(x)$ is the Heaviside step function.

The symmetry group of graphene lattice is $D_{6 h}$, so that $\chi_{g}^{(3)}$ has only two independent non-zero components: $\chi_{g, x x x x}^{(3)}=$ $\chi_{g, y y y y}^{(3)}$ and $\chi_{g, x y x y}^{(3)}=\chi_{g, x x y y}^{(3)}=\chi_{g, x y y x}^{(3)}=\chi_{g, y x x y}^{(3)}=\chi_{g, y x y x}^{(3)}=$ $\chi_{g, y y x x}^{(3)}$. The dominant component is $\chi_{g, x x x x}^{(3)} \equiv \chi_{g}^{(3)}$, and is depicted by dashed curves in Fig. 4. Importantly, it can be readily seen from Eq. (10) that $\bar{\chi}_{i j k l}^{(3)}$ and $\chi_{g, i j k l}^{(3)}$ have the same set of non-zero components, so that the effective third-order susceptibility has only two independent non-zero components, too: $\bar{\chi}_{x x x x}^{(3)}=\bar{\chi}_{y y y y}^{(3)} \equiv \bar{\chi}_{1}^{(3)}$ and $\bar{\chi}_{x y x y}^{(3)} \equiv \bar{\chi}_{2}^{(3)}$. In order to quantify the enhancement of the nonlinear optical response of the graphene metasurface, we also computed the ratio $\eta=\left|\bar{\chi}_{1}^{(3)}\right| /\left|\chi_{g}^{(3)}\right|$.

The results presented in Fig. 4 reveal several important conclusions. First, the dominant component of $\bar{\chi}^{(3)}$ is $\bar{\chi}_{x x x x}^{(3)}$, but unlike the monotonous frequency dependence of graphene thirdorder susceptibility, the frequency dependence of $\bar{\chi}_{x x x x}^{(3)}$ suggests a resonant nonlinear optical response. As in the linear case, the resonances of the optical nonlinearity of the homogenized metasurface coincide with the plasmon-induced peaks shown in Fig. 2. Second, due to these plasmon-induced resonances, the effective third-order susceptibility of the graphene metasurface is strongly enhanced as compared to that of a graphene sheet; e.g., at the wavelength $\lambda_{T H}=5.4 \mu \mathrm{m}$ of the main resonance the enhancement factor $\eta$ is about two orders of magnitude.

In order to validate the nonlinear part of the proposed homogenization method, the THG of both the graphene cruciform metasurface and the corresponding homogeneous layer of material have been calculated using an in-house developed generalizedsource-FDTD code [29]. The results of these computations are presented and compared in Fig. 5, where the corresponding THG spectra are plotted. It can be seen from this figure that there is a very good agreement between the two spectra, a maximum difference of about $5 \%$ being observed at the wavelength of the main resonance. Moreover, the very good agreement regarding both linear and nonlinear response of the graphene metasurface and its homogenized counterpart, illustrated in Fig. 3 and Fig. 5, respectively, indicate that a patterned graphene metasurface can be accurately replaced with a simple homogenous layer of material with an effective permittivity and nonlinear susceptibility retrieved with our homogenization method.

In summary, we have introduced a versatile and efficient homogenization method for the study of the linear and nonlinear optical response of graphene metasurfaces. Our study shows that the third-order nonlinearity of such metasurfaces is enhanced by more than two order of magnitude at the frequencies of surface plasmons of the graphene components of the metasurface. Due to its versatility, our method can be extended to metasurfaces containing two-dimensional materials other than graphene, to three-dimensional metamaterials, and to a large class of nonlinear optical interactions. Due to all these powerful features, our method has great potential to facilitate the design of active photonic devices with advanced functionalities. Funding. European Research Council (ERC-2014-CoG-648328).

\section{REFERENCES}

1. K. S. Novoselov, A. K. Geim, S. V. Morozov, D. Jiang, Y. Zhang, S. V. Dubonos, I. V. Grigorieva, and A. A. Firsov, Science 306, 666 (2004).

2. F. N. Xia, W. Han, X. Di, D. Madan, and R. Ashwin, Nat. Photonics 8, 899 (2014).

3. H. Yan, T. Low, W. Zhu, Y. Wu, M. Freitag, X. Li, F. Guinea, P. Avouris, and F. Xia, Nat. Photonics 7, 394 (2013).

4. T. Low, A. Chaves, J. D. Caldwell, A. Kumar, N. X. Fang, P. Avouris, T. F. Heinz, F. Guinea, L. Martin-Moreno, and F. Koppens, Nat. Mater. 16, 182 (2017).

5. A. K. Geim, Science 324, 1530 (2009).

6. G. Fiori, F. Bonaccorso, G. Iannaccone, T. Palacios, D. Neumaier, A. Seabaugh, S. K. Banerjee, and L. Colombo, Nat. Nanotechnol. 9, 768 (2014).

7. G. S. Kulkarni, K. Reddy, Z. Zhong, and X. Fan, Nat. Commun. 5, 4376 (2014).

8. M. Zhong, D. Xu, X. Yu, K. Huang, X. Liu, Y. Qu, Y. Xu, and D. Yang, Nano Energy 28, 12 (2016)

9. M. M. Jadidi, J. C. Konig-Otto, S. Winnerl, A. B. Sushkov, H. D. Drew, T. E. Murphy, and M. Mittendorff, Nano Lett. 16, 2734 (2016).

10. G. K. Lim, Z. L. Chen, J. Clark, R. G. Goh, W. H. Ng, H. W. Tan, R. H. Friend, P. K. Ho, and L. L. Chua, Nat. Photonics 5, 554 (2011).

11. M. L. Nesterov, J. Bravo Abad, A. Y. Nikitin, F. J. Garcia Vidal, and L. Martin Moreno, Laser Photon. Rev. 7, 7 (2013).

12. H. Deng, F. Ye, B. A. Malomed, X. Chen, and N. C. Panoiu, Phys. Rev. B 91, 201402 (2015).

13. J. W. You, S. R. Bongu, Q. Bao, and N. C. Panoiu, Nanophotonics 8, 63 (2019).

14. A. Andryieuski and A. V. Lavrinenko, Opt. Express 21, 9144 (2013).

15. J. W. You, J. You, M. Weismann, and N. C. Panoiu, Phil. Trans. R. Soc. A 375, 20160313 (2017).

16. Y. Zhang, Y. Feng, B. Zhu, J. Zhao, and T. Jiang, Opt. Express 23, 27230 (2015).

17. J. W. You and N. C. Panoiu, Opt. Express 26, 1882 (2018).

18. Y. Fan, Z. Wei, Z. Zhang, and H. Li, Opt. Lett. 38, 5410 (2013).

19. Y. Fan, N. H. Shen, F. Zhang, Q. Zhao, Z. Wei, P. Zhang, J. Dong, Q. Fu, H. Li, and C. M. Soukoulis, ACS Photonics 5, 1612 (2018).

20. M. Mayer, M. J. Schnepf, T. A. Konig, and A. Fery, Adv. Opt. Mater. 7, 1800564 (2019).

21. D. R. Smith, S. Schultz, P. Markos, and C. M. Soukoulis, Phys. Rev. B 65, 195104 (2002).

22. D. R. Smith and J. B. Pendry, J. Opt. Soc. Am. B 23, 391 (2006).

23. S. Larouche and D. R. Smith, Opt. Commun. 283, 1621 (2010).

24. M. A. Gorlach, T. A. Voytova, M. Lapine, Y. S. Kivshar, and P. A. Belov, Phys. Rev. B 93, 165125 (2016).

25. P. A. D. Goncalves and N. M. Peres, An introduction to graphene plasmonics (World Scientific, 2016).

26. V. Lucarini, J. J. Saarinen, K. E. Peiponen, and E. M, Vartiainen, Kramers-Kronig relations in optical materials research (Springer, 2005).

27. R. M. Roth, N. C. Panoiu, M. M. Adams, J. I. Dadap, and R. M. Osgood, Opt. Lett. 32, 3414 (2007).

28. J. D. Cox, I. Silveiro and F. J. G. de Abajo, ACS Nano 10, 1995 (2016).

29. J. W. You, E. Threlfall, D. F. G. Gallagher, and N. C. Panoiu, J. Opt. Soc. Am. B 35, 2754 (2018). 


\section{REFERENCES}

1. K. S. Novoselov, A. K. Geim, S. V. Morozov, D. Jiang, Y. Zhang, S. V. Dubonos, I. V. Grigorieva, and A. A. Firsov, "Electric field effect in atomically thin carbon films," Science 306, 666 (2004).

2. F. N. Xia, W. Han, X. Di, D. Madan, and R. Ashwin, "Two-dimensional material nanophotonics," Nat. Photonics 8, 899 (2014).

3. H. Yan, T. Low, W. Zhu, Y. Wu, M. Freitag, X. Li, F. Guinea, P. Avouris, and $\mathrm{F}$. Xia, "Damping pathways of mid-infrared plasmons in graphene nanostructures," Nat. Photonics 7, 394 (2013).

4. T. Low, A. Chaves, J. D. Caldwell, A. Kumar, N. X. Fang, P. Avouris, T. F. Heinz, F. Guinea, L. Martin-Moreno, and F. Koppens, "Polaritons in layered two-dimensional materials," Nat. Mater. 16, 182 (2017).

5. A. K. Geim, "Graphene: status and prospects," Science 324, 1530 (2009).

6. G. Fiori, F. Bonaccorso, G. lannaccone, T. Palacios, D. Neumaier, A. Seabaugh, S. K. Banerjee, and L. Colombo, "Electronics based on two-dimensional materials," Nat. Nanotechnol. 9, 768 (2014).

7. G. S. Kulkarni, K. Reddy, Z. Zhong, and X. Fan, "Graphene nanoelectronic heterodyne sensor for rapid and sensitive vapour detection," Nat. Commun. 5, 4376 (2014).

8. M. Zhong, D. Xu, X. Yu, K. Huang, X. Liu, Y. Qu, Y. Xu, and D. Yang, "Interface coupling in graphene/fluorographene heterostructure for highperformance graphene/silicon solar cells," Nano Energy 28, 12 (2016).

9. M. M. Jadidi, J. C. Konig-Otto, S. Winnerl, A. B. Sushkov, H. D. Drew, T. E. Murphy, and M. Mittendorff, "Nonlinear terahertz absorption of graphene plasmons," Nano Lett. 16, 2734 (2016).

10. G. K. Lim, Z. L. Chen, J. Clark, R. G. Goh, W. H. Ng, H. W. Tan, R. H. Friend, P. K. Ho, and L. L. Chua, "Giant broadband nonlinear optical absorption response in dispersed graphene single sheets," Nat. Photonics 5, 554 (2011).

11. M. L. Nesterov, J. Bravo Abad, A. Y. Nikitin, F. J. Garcia Vidal, and L. Martin Moreno, "Graphene supports the propagation of subwavelength optical solitons," Laser Photon. Rev. 7, 7 (2013).

12. H. Deng, F. Ye, B. A. Malomed, X. Chen, and N. C. Panoiu, "Optically and electrically tunable Dirac points and Zitterbewegung in graphenebased photonic superlattices," Phys. Rev. B 91, 201402 (2015).

13. J. W. You, S. R. Bongu, Q. Bao, and N. C. Panoiu, "Nonlinear optical properties and applications of $2 \mathrm{D}$ materials: theoretical and experimental aspects," Nanophotonics 8, 63 (2019).

14. A. Andryieuski and A. V. Lavrinenko, "Graphene metamaterials based tunable terahertz absorber: effective surface conductivity approach," Opt. Express 21, 9144 (2013).

15. J. W. You, J. You, M. Weismann, and N. C. Panoiu, "Double-resonant enhancement of third-harmonic generation in graphene nanostructures," Phil. Trans. R. Soc. A 375, 20160313 (2017).

16. Y. Zhang, Y. Feng, B. Zhu, J. Zhao, and T. Jiang, "Switchable quarterwave plate with graphene based metamaterial for broadband terahertz wave manipulation," Opt. Express 23, 27230 (2015).

17. J. W. You and N. C. Panoiu, "Polarization control using passive and active crossed graphene gratings," Opt. Express 26, 1882 (2018).

18. Y. Fan, Z. Wei, Z. Zhang, and H. Li, "Enhancing infrared extinction and absorption in a monolayer graphene sheet by harvesting the electric dipolar mode of split ring resonators," Opt. Lett. 38, 5410 (2013).

19. Y. Fan, N. H. Shen, F. Zhang, Q. Zhao, Z. Wei, P. Zhang, J. Dong, Q. Fu, H. Li, and C. M. Soukoulis, "Photoexcited graphene metasurfaces: significantly enhanced and tunable magnetic resonances," ACS Photonics 5, 1612 (2018).

20. M. Mayer, M. J. Schnepf, T. A. Konig, and A. Fery, "Colloidal SelfAssembly Concepts for Plasmonic Metasurfaces," Adv. Opt. Mater. 7, 1800564 (2019).

21. D. R. Smith, S. Schultz, P. Markos, and C. M. Soukoulis, "Determination of effective permittivity and permeability of metamaterials from reflection and transmission coefficients," Phys. Rev. B 65, 195104 (2002).

22. D. R. Smith and J. B. Pendry, "Homogenization of metamaterials by field averaging," J. Opt. Soc. Am. B 23, 391 (2006).

23. S. Larouche and D. R. Smith, "A retrieval method for nonlinear metamaterials," Opt. Commun. 283, 1621 (2010).

24. M. A. Gorlach, T. A. Voytova, M. Lapine, Y. S. Kivshar, and P. A. Belov,
"Nonlocal homogenization for nonlinear metamaterials," Phys. Rev. B 93, 165125 (2016).

25. P. A. D. Goncalves and N. M. Peres, An introduction to graphene plasmonics (World Scientific, 2016).

26. V. Lucarini, J. J. Saarinen, K. E. Peiponen, and E. M, Vartiainen, Kramers-Kronig relations in optical materials research (Springer, 2005).

27. R. M. Roth, N. C. Panoiu, M. M. Adams, J. I. Dadap, and R. M. Osgood, "Polarization-tunable plasmon-enhanced extraordinary transmission through metallic films using asymmetric cruciform apertures," Opt. Lett. 32, 3414 (2007).

28. J. D. Cox, I. Silveiro and F. J. G. de Abajo, "Quantum effects in the nonlinear response of graphene plasmons," ACS Nano 10, 1995 (2016).

29. J. W. You, E. Threlfall, D. F. G. Gallagher, and N. C. Panoiu, "Computational analysis of dispersive and nonlinear 2D materials by using a GS-FDTD method," J. Opt. Soc. Am. B 35, 2754 (2018). 